\title{
The Use of Transvaginal Ultrasound by Emergency Physicians in Medical Student Education
}

\author{
${ }^{1}$ Nadah B Zafar, ${ }^{2}$ Veronica Greer, ${ }^{3}$ Robert H Woolard \\ ${ }^{1}$ Assistant Professor, Department of Medical Education, Clinical Assistant Professor of Emergency Medicine, Paul L Foster \\ School of Medicine, Texas Tech University Health Sciences Center, 5001 El Paso Drive, EI Paso, Texas 79905, USA \\ ${ }^{2}$ Assistant Professor and Residency Director, Department of Emergency Medicine, Texas Tech University Health Sciences \\ Center, Paul L Foster School of Medicine, 4800 Alberta Avenue, El Paso, Texas 79905, USA \\ ${ }^{3}$ Professor, Department of Emergency Medicine, Texas Tech University Health Sciences Center, Paul L Foster School of \\ Medicine, 4800 Alberta Avenue, El Paso, Texas 79905. USA
}

Correspondence: Nadah B Zafar, Assistant Professor, Department of Medical Education, Clinical Assistant Professor of Emergency Medicine, Paul L Foster School of Medicine, Texas Tech University Health Sciences Center, 5001 El Paso Drive, El Paso, Texas 79905, USA, Phone: (915) 783-1700 Ext. 229, Fax: (915) 783-1715, e-mail: nadah.zafar@ttuhsc.edu

\begin{abstract}
Paul L Foster School of Medicine at Texas Tech University Health Sciences Center in El Paso offers the students a faculty (both clinician and basic scientists) guided clinical presentation based curriculum. Emergency physicians have been an integral part of this curriculum. Bedside transvaginal ultrasound has become an adjunct to the history and physical examination for the evaluation in a timely manner of acute pelvic and lower abdominal pain and vaginal bleeding in the female of reproductive years. Discussion of the approach to diagnosis is divided into two broad categories; evaluation when there's a positive pregnancy test (first trimester) and a negative pregnancy test. The discussion also illustrates how the emergency physician can, using ultrasound, introduce, integrate, and review, the pertinent basic sciences (anatomy, embryology, pathology, physiology, microbiology and biochemistry) with the medical student.
\end{abstract}

Keywords: Transvaginal pelvic ultrasound, pelvic pain, vaginal bleeding, first trimester, medical education, gynecological emergencies.

\section{INTRODUCTION}

In emergency medicine bed side ultrasound has been a monumental addition for the early detection of life-threatening disorders. Emergency physicians routinely perform a limited goal directed bedside ultrasound evaluation of their patients. ${ }^{1}$ Within the first two years of the integrated, clinical presentation based medical curriculum at the Paul L Foster School of Medicine at Texas Tech University Health Science Center in El Paso, emergency medicine faculty have found ultrasound to be an excellent adjunct when teaching clinical assessment and physical examination.

Females presenting with pelvic and lower abdominal pain with or without vaginal bleeding "pelvic pain" are common to emergency practice. Early detection of gynecological emergencies like ectopic pregnancies is paramount to the emergency physician. Pelvic ultrasound is integral to the practice of many emergency physicians as an extension of the physical examination. ${ }^{2}$ In many situations addressing the stability of the patient occurs simultaneously with arriving at a diagnosis. "The use of ultrasound should never preclude adequate resuscitation or definitive surgical therapy in a patient who is hemodynamically unstable." ${ }^{3}$ Emergency medicine faculty when introducing these clinical presentations to the student can better integrate concepts from the basic sciences: anatomy, biochemistry embryology, pathology and microbiology by incorporating findings from goal directed ultrasound.

When guiding the student on the evaluation of "pelvic pain" the clinician should explain their thought processes; a first step is to place the patient into one of these categories: premenarchal, reproductive years and perimenopausal. This discussion will focus on the use of transvaginal ultrasound by the emergency physician to evaluate females of reproductive years and how findings from ultrasound can reinforce for the student integration of the concepts from basic science. After the emergency physician establishes that the patient with "pelvic pain" is in their reproductive years, the next step is determining pregnancy status which is by done by obtaining either a urine or serum a qualitative or quantitative human chorionic gonadotropin (hCG). The student now can be taught more about hCG, a glycoprotein produced by the trophoblast after implantation (biochemistry 
and embryology). The approach to the patient will fall onto one of two paths depending upon the results of the pregnancy test.

\section{ACUTE PELVIC AND LOWER ABDOMINAL PAIN WITH OR WITHOUT VAGINAL BLEEDING "PELVIC PAIN" IN THE PREGNANT PATIENT}

When performing the history and physical examination the student may encounter signs of pregnancy. To better understand the concept of normal pregnancy, the student will be encouraged to establish which trimester the patient is by dates, examination including ultrasound and hCG levels. In the United States, for the most part in academic centers, women over $\geq 20$ weeks of gestation with "pelvic pain" are initially seen by the department of obstetrics unless the patient is in an immediate life-threatening situation or trauma is involved in the clinical presentation. Hence the emergency physician is usually evaluating first trimester or early second trimester patients.

\section{ACUTE "PELVIC PAIN" IN THE PREGNANT PATIENT}

\section{First Trimester: Ectopic Pregnancy}

All clinicians must consider the diagnosis of ectopic pregnancy with first trimester pelvic and lower abdominal pain with or without vaginal bleeding. When the diagnosis of ectopic pregnancy is considered the emergency physician can naturally direct the medical student to the review of pertinent normal and abnormal fetomaternal physiology, embryology and the pathology.

Ectopic pregnancy is the most common cause of pregnancy related death in the first trimester and accounts for about $10 \%$ of all pregnancy related deaths. ${ }^{3}$ Missed ectopic pregnancy is a leading cause of emergency medicine malpractice claims. ${ }^{3}$ The classic symptoms of abdominal pain with vaginal bleeding and spotting and delayed menses does not commonly occur. As the history is highly variable so is the physical examination. The patient can have a spectrum of minimal to severe pain, no vaginal blood to gross vaginal bleeding, little to severe tenderness and vital signs ranging from normal to hypotension and shock.

Transabdominal and transvaginal are acceptable modalities to evaluate pelvic pathology but the advantage of transvaginal technique for the emergency physician is that it does not require a full bladder. In addition, palpation of the pelvic area with transvaginal technique will allow better identification of fixed structures like the ovaries because during transvaginal imaging an obscuring loop of intestines can be pushed out of the way. ${ }^{4}$ In addition, transvaginal ultrasound can detect an intrauterine pregnancy at 4.5-5 weeks as opposed to a transabdominal ultrasound which detects pregnancy at 6 weeks. ${ }^{4}$

The ultrasound aids the student in review of normal female gynecological anatomy and the anatomy of nearby structures (i.e. the position of the bladder in relation to the uterus, and the uterus in relation to the adnexa, and cervix). In early pregnancy the goal of the emergency physician performing a bedside ultrasound on a patient with pelvic pain is to detect an intrauterine pregnancy (IUP). An ectopic pregnancy can be virtually excluded by the presence of an IUP on transvaginal ultrasound as heterotrophic pregnancy is very rare in patients who are not undergoing fertility treatment. ${ }^{4} \mathrm{~A}$ limited goal directed ultrasound is different than the formal ultrasound performed in the department of diagnostic imaging. As an aside, in many European countries transvaginal ultrasound is only done by obstetricians and gynecologists.

The physician can explain to the student the use of a quantitative hCG in conjunction with ultra sonography, introducing the concept of the discriminatory zone. The discriminatory zone represents the level of hCG above which an intrauterine pregnancy should be detected on ultrasound. The general consensus for goal directed emergency transabdominal ultrasound is that at a level of $\geq 1500 \mathrm{mlU} /$ $\mathrm{ml}$ an IUP should be detectable. ${ }^{3,4}$ The discriminatory zone depends upon the ultrasound technique, technology and approach; it may be as low as $1000 \mathrm{mlU} / \mathrm{ml}$ for a transvaginal ultrasound (TVS). ${ }^{3-5}$ "The earliest sonographic sign of an intrauterine pregnancy is the presence of a true gestational sac, which has double echogenic rings (double decidual sack sign) surrounding the sac (created by hypoechoic fluid between the decidua parietalis and the decidua capsularis)" which is usually visible at 4.5 to 5 weeks using a TVS. ${ }^{6}$ Although the earliest sign of an intrauterine pregnancy is a true gestational sac, the emergency physician must identify the yolk sac appearing at 5 to 6 weeks to confirm an IUP., When only a double decidual sack sign is found, there remains a possibility that the finding is a pseudogestational sac. ${ }^{4,6}$ Cardiac activity is usually detected at 5.5 to 6 weeks. ${ }^{6}$ For the medical student visualization of any of these items (gestational sac, yolk sac, and cardiac activity) prompts a discussion of embryology and which can be viewed in real time, bringing basic science to life. 
Finding an intrauterine pregnancy virtually rules out the diagnosis of an ectopic pregnancy as long as there is a there is a single gestation. ${ }^{3,4,6,7}$

If the emergency physician demonstrates an empty uterus and embryonic cardiac activity outside the uterus diagnosis of ectopic pregnancy can be made. ${ }^{4,7}$ If a complex adnexal mass is seen in conjunction with free fluid in the pelvis an ectopic pregnancy is highly likely. ${ }^{4,5,7}$ As mentioned previously the emergency bedside ultrasound is a limited focused ultrasound. Any findings other than sonographically demonstrated intrauterine pregnancy require a formal ultrasound by the department of diagnostic imaging and consultation with an obstetrician/gynecologist. ${ }^{1,4,7}$

Common ultrasound criteria (both uterine and extrauterine) for the diagnosis of ectopic pregnancy are:

- "Empty uterus (with serum beta hCG $\geq 1500 \mathrm{mlU} / \mathrm{mLs}$ ) or a uterine cavity with increased endometrial thickness, central hypoechoic areas or a sac like structure (pseudogestational sac)". 5

- Visualization of cardiac activity, or a true gestation sac, yolk sac and fetal pole outside the uterus ${ }^{5,6}$ (Figs 1 and 2).

Other sonographic findings which are highly suggestive of an ectopic pregnancy:

- Presence of tubal ring in the adnexa and or the presence of free fluid in the pelvis with a complex adnexal mass. ${ }^{5,6}$

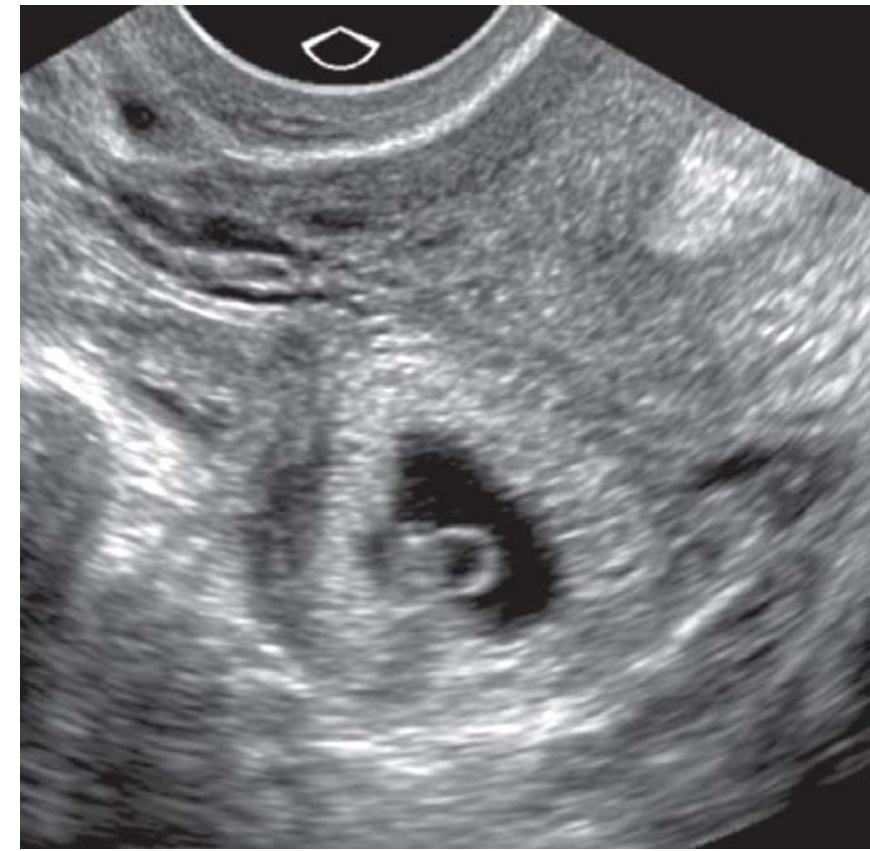

Fig. 1: Transvaginal scan of a gestational sac in the adnexal region. Yolk sac and embryonic pole are clearly visualized

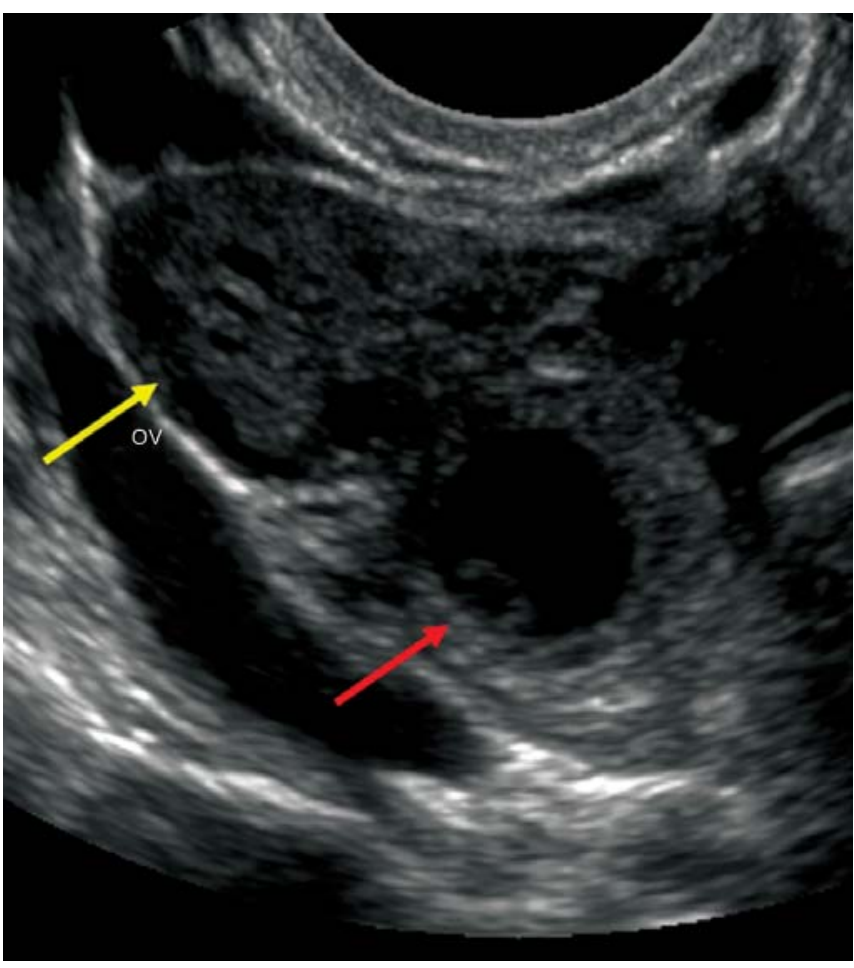

Fig. 2: Transvaginal scan demonstrating a concentric gestational ring with yolk sac in the adnexal region (red arrow). Ipsilateral ovary is demonstrated laterally (yellow arrow)

- Presence of a complex adnexal mass: Very often an unspecified adnexal tumor could be visualized. "Color flow Doppler is an excellent and rapid guide for identification of the peritrophoblastic tissue in patients with an ectopic pregnancy to distinguish it from an adnexal mass." 5 (Fig. 3).

When teaching the medical student, faculty can use the above ultrasound findings to beautifully illustrate gynecological anatomy, abnormal embryological development and pathology which can be integrated with biochemistry $(\beta \mathrm{hCG})$ in the process of evaluating an ectopic pregnancy.

\section{ACUTE PELVIC/ABDOMINAL PAIN AND OR VAGINAL BLEEDING IN THE PREGNANT PATIENT}

\section{First Trimester: Other Diagnosis}

Once ectopic pregnancy is definitely ruled in or out other diagnosis to consider with "pelvic pain" in a first trimester pregnant woman are:

1. Threatened abortion.

2. Inevitable abortion. 


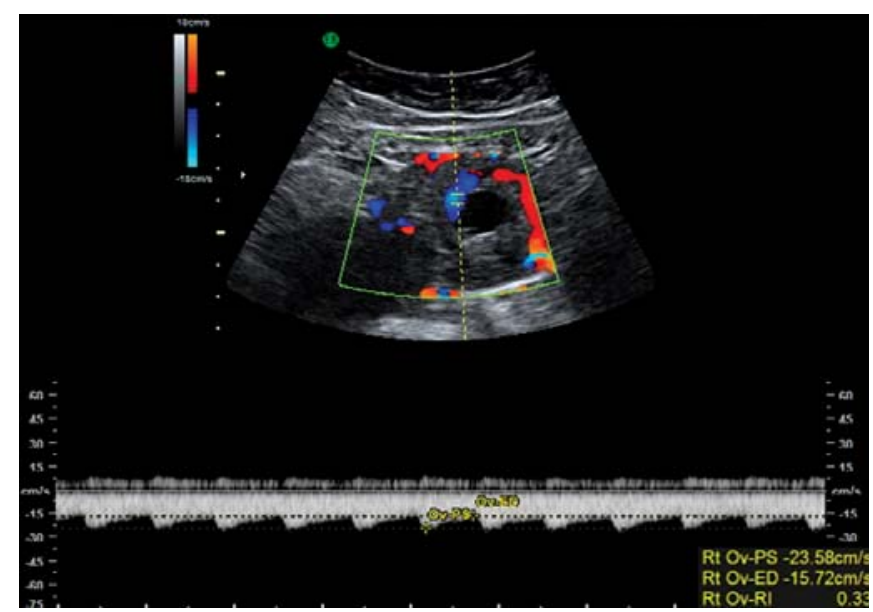

Fig. 3: Transvaginal color Doppler scan of ectopic pregnancy. Pulsed Doppler waveform analysis demonstrates low vascular resistance (RI of 0.33 indicating prominent trophoblastic vitality and invasiveness

3. Early intrauterine pregnancy: When the ultrasound shows an empty uterus with a serum hCG $<1500 \mathrm{mlU} /$ $\mathrm{mL}$, the patient can be observed and have serial hCG measurements every 2 days with a repeat of the transvaginal ultrasound once the levels are $\geq 1500 \mathrm{mlU} /$ $\mathrm{mL} \cdot{ }^{4,7}$ If hCG failed to rise by a level of at least $66 \%$ in a two days period an operative procedure is used to diagnose ectopic pregnancy. ${ }^{4,7}$

4. Failed intrauterine pregnancy:

- Blighted ovum ${ }^{7}$.

- Grossly deformed gestational sac visualized on ultrasound almost always predicts fetal demise (Figs $4 \mathrm{~A}$ to $\mathrm{C})^{7}$

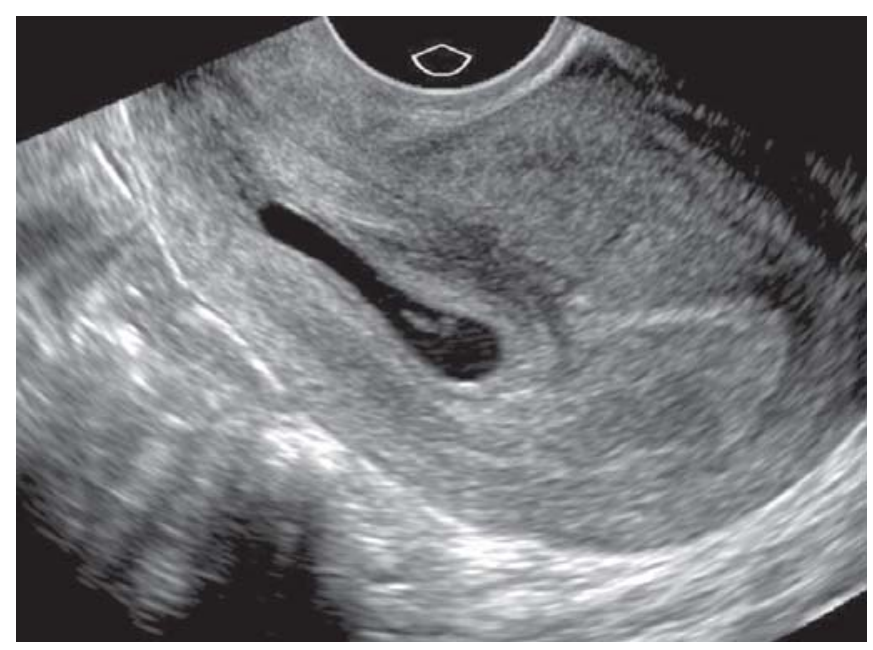

Fig. 4A: Transvaginal scan of irregular gestational sac in the lower uterine segment

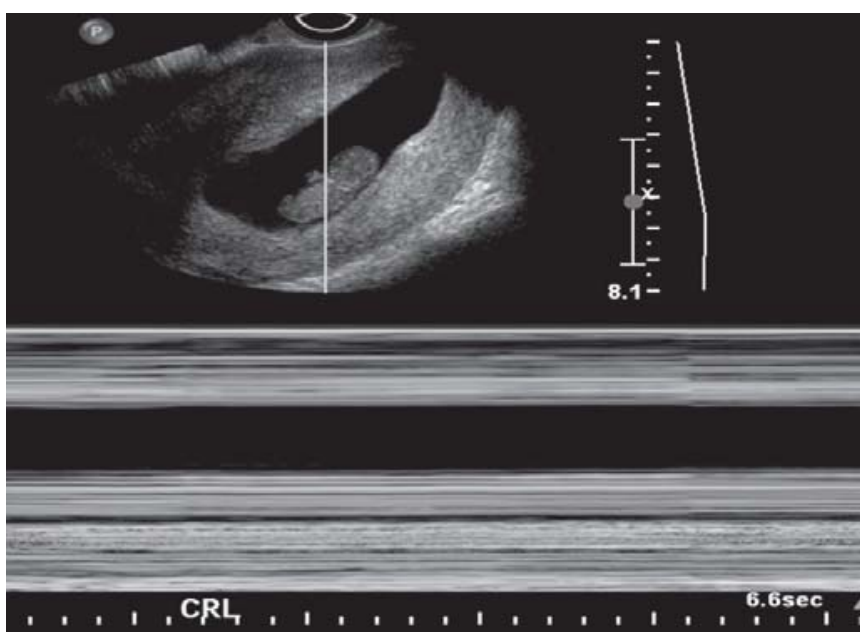

Fig. 4B: M-mode demonstrates absence of embryonic heart activity

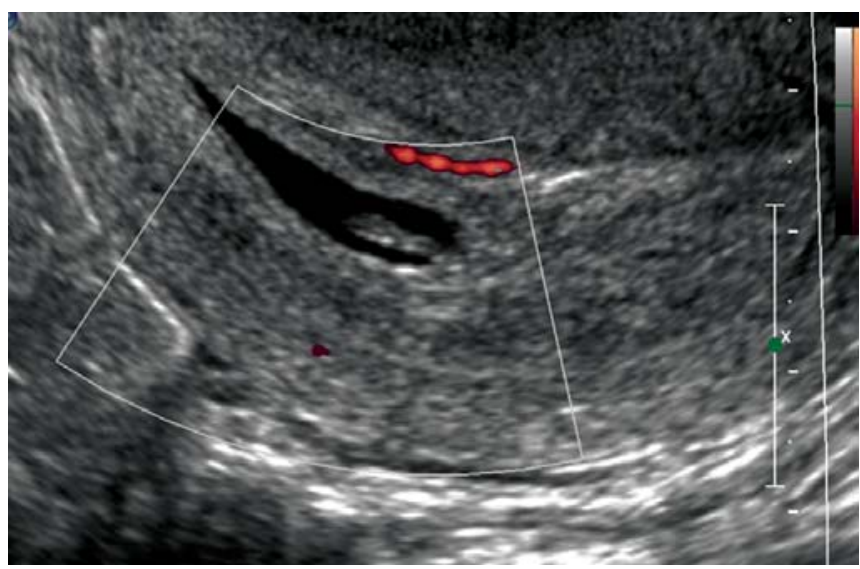

Fig. 4C: Power Doppler ultrasound of the same patient. Blood flow signals are obtained from the spiral arteries, while absence of heart activity is noted by power Doppler

- A gestational sac located in the lower portion of the uterus adjacent to the cervix is most likely in the process of aborting. ${ }^{7}$

5. Subchorionic hematoma.

6. Spontaneous abortion.

For the medical student, these ultrasound findings can integrate the learning of embryology, biochemistry and pathology with specific clinic presentations.

\section{ACUTE "PELVIC PAIN" WITH A NEGATIVE PREGNANCY TEST}

The spectrum of disease is broad with this category. This discussion is focusing on gynecological emergencies. Pathologies to consider are ruptured ovarian cyst, adnexal/ ovarian torsion and pelvic inflammatory disease (PID) 


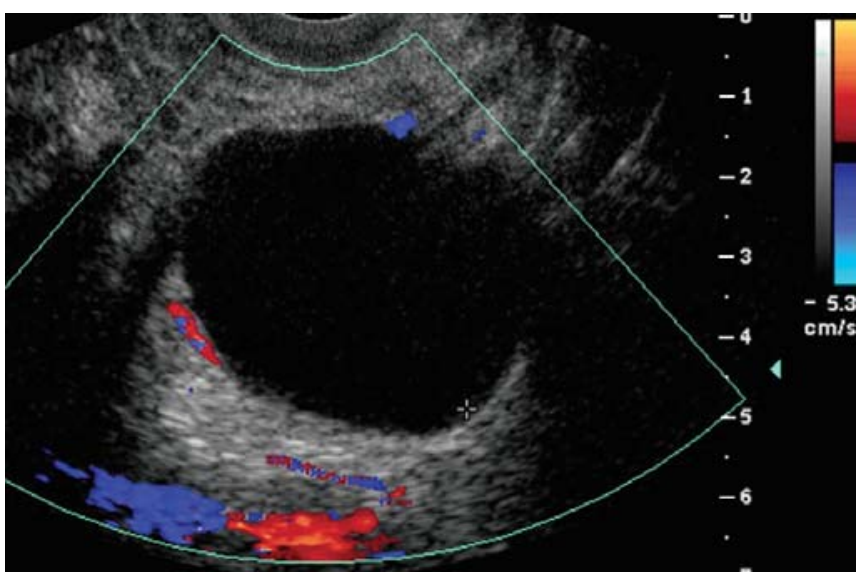

Fig. 5: Transvaginal color Doppler ultrasound of a unilocular sonolucent cystic structure. Peripheral blood flow signals virtually exclude adnexal torsion

complicated with a tubo-ovarian abscess. Again a transvaginal ultrasound in conjunction with the history and physical examination aids the emergency physician in the timely diagnosis of these conditions.

A normal ovary is $2.5-5 \mathrm{~cm}$ long, $1.5-3 \mathrm{~cm}$, wide and 61-1.5 cm thick. ${ }^{8}$ On sonogram ovarian cysts have a thin rounded wall and a unilocular appearance that is either is hypoechoic or anechoic. ${ }^{5,8}$ Ultrasound demonstration of an ovarian cyst will prompt review of pathology of cysts and normal anatomy of ovaries (Fig. 5). "Corpus luteum cysts may be painful either due to the large size of the cyst, or due to the hemorrhage within the cysts." ${ }^{5}$ Here again demonstrating a corpus luteum cyst will prompt review of hormonal physiology.

Torsion of the ovaries and fallopian tubes is an uncommon but serious gynecological emergency and usually occurs due to a pathologically enlarged ovary $>4 \mathrm{~cm}$ in diameter. ${ }^{5}$ Ultrasound is the primary imaging modality used when ovarian torsion is suspected. Impairment of venous and lymphatic drainage from the torsion leads to an enlarged ovary which is the most common sonographic finding for an ovarian torsion. ${ }^{8}$ Combination of color flow Doppler imaging and assessment of the ovary increases diagnostic accuracy and early diagnosis of torsion before irreversible ischemic changes occur., "In the initial stages of torsion, venous blood flow is reduced, arterial signals demonstrate high impedance blood flow signals indicating that occlusion of both venous and lymphatic systems occur first." ${ }^{5}$ At a later stage, when there is total occlusion involving the arterial circulation, no adnexal blood flow is seen. ${ }^{5}$ Since the complication of necrosis and ischemia is ever present the student here has the opportunity to review the vascular supply and lymphatic drainage of the ovaries in addition to the pathophysiology of ischemia and infarction.

PID is caused by organisms ascending the upper female genital tract from the vagina. This disease ranges from endometritis, salpingitis, and tubo-ovarian abscess. ${ }^{9}$ The diagnosis of PID is usually made clinically. Ultrasound may not be necessary in uncomplicated cases and may be normal early in the disease. It is usually ordered in severe cases of PID to diagnose a tubo-ovarian abscess.

Ultrasound findings in PID may include the following:

- Fluid in the cul-de-sac., ${ }^{4,5}$

- Thickening of the tube wall $\geq 5 \mathrm{~mm}$.,5

- Pyosalpinx which appears sonographically as circular structure with low level echoes in the lumen when viewed in cross-section. ${ }^{4,5}$

- "Incomplete septations and beads on a sting sign which represents hyperechoic mural nodules measuring approximately 2 to $3 \mathrm{~mm}$, visualized on the cross-section of a fluid filled distended tube" (Fig. 6). ${ }^{5}$

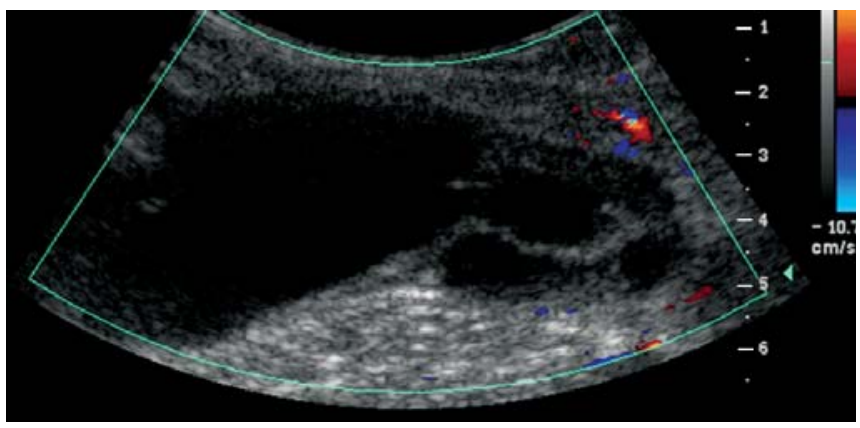

Fig. 6: Complex adnexal mass with incomplete septations represents a fluid filled distended tube in a patient with chronic PID. Discrete blood flow signals within the tubal walls provide additional information in the diagnosis of PID

- An abscess will appear as a tubo-ovarian complex mass (the ovaries cannot be separated from the tube by pushing the mass with the vaginal probe). ${ }^{5}$

- This mass may have both cystic and solid components. ${ }^{4}$ Again, clinical presentations including ultrasound can aid the medical student when learning normal anatomy, pathology and microbiology (PID).

\section{CONCLUSION}

In combination with history, and physical examination, the use of transvaginal and transabdominal ultrasound helps the emergency physician to accurately and in a timely manner diagnose and manage both acute gynecological and obstetrical emergencies in the female of reproductive 
years. ${ }^{1,3}$ In addition, the emergency physician, when teaching medical students about the clinical presentation and evaluation of acute "pelvic pain" (pelvic and lower abdominal pain with or without vaginal bleeding) can use ultrasound on as an adjunct to the history and physical examination, to integrate the relevant basic science concepts from anatomy, pathology, physiology, microbiology and biochemistry. Clinical presentations and ultrasonic images can provide memorable details that demonstrate the application of basic concepts.

\section{ACKNOWLEDGMENT}

All of the images for this article were provided by Sanja P. Kupesic, MD, PhD, Professor of Obstetrics and Gynecology in the Department of Medical Education at the Paul L Foster School of Medicine in El Paso, Texas.

\section{REFERENCES}

1. Burgher SW, Tandy TK, Dawdy MR. Transvaginal Ultrasonography by Emergency Physicians Decreases Patient Time in the Emergency Department. Acad Emerg Med Aug 1998;5:802-07.
2. Valley VT, Fly CA. "Ultrasonography, Pelvic". Available on line: Http://.emedicine.medscape.com. (Downloaded: September 3, 2009).

3. Jang T, Chen JC. "Bedside Ultrasonography, First -Trimester". Available on line: Http://www. emedicine.medscape.com. (Downloaded: September 8, 2009).

4. Reardon RF, Jehle DVK. Pelvic Ultrasonography. In: Tintinalli JE, Kelen GD, Strapczynski SJ. Emergency Medicine a Comprehensive Study Guide (6th ed). USA: McGraw Hill Companies, Inc. 2008;113:715-26.

5. Kupesic S, Plavsic BM. Sonographic Evaluation of Gynecological and Obstetric Causes of Acute Pelvic Pain. In: Kurjak A, Chervenak FA. Donald School Textbook of Ultrasound in Obstetrics and Gynecology. New Delhi India, United Kingdom: The Parthenon Publishing Group 2006;63;939-50.

6. Tulandi T. "Clinical manifestations, diagnosis and management of ectopic pregnancy". Available on line: Http:// www.uptodate.com. (Downloaded September 8, 2009).

7. Krause RS, Janicke DM. Ectopic Pregnancy. In: Tintinalli JE, Kelen GD, Strapczynski SJ. Emergency Medicine a Comprehensive Study Guide (6th ed). USA: McGraw Hill Companies, Inc. 2008, Chapter 103:658-64.

8. Valesky Jr. WW, Silverberg MA. "Ovarian Cysts". Available on line: Http://.emedicine.medscape.com. (Downloaded: September 11, 2009).

9. Reyes I, Kumar R. "Pelvic Inflammatory disease". Available on line: Http://.emedicine.medscape.com. (Downloaded: September 11, 2009). 